\title{
Apoiando a Avaliação do Desempenho dos Alunos com Base em Jogos Educacionais
}

\author{
Handerson Bezerra Medeiros ${ }^{1}$, Eduardo Henrique da Silva Aranha ${ }^{1}$ \\ ${ }^{1}$ Departamento de Informática e Matemática Aplicada (DIMAP) \\ Universidade Federal do Rio Grande do Sul (UFRN) \\ handerson.medeiroseimd.ufrn.br, eduardoaranha@dimap.ufrn.br
}

\begin{abstract}
Although educational games can improve student performance, teachers should have information about student performance in order to better conduct their classes or to give personalized assistance, according to the needs of each student. To support these performance analyses, this work proposes an approach that is structured as a supporting platform and a game based educational software. By using our approach, educational games can not only promote benefits for the students but also give relevant information about student performance (common mistakes, step-by-step simulation, competencies, and abilities, etc.), helping teachers in their academic activities.
\end{abstract}

Resumo. Embora os jogos educativos possam melhorar o desempenho dos alunos, os professores devem ter informações sobre o desempenho dos alunos, a fim de conduzir melhor suas aulas ou prestar uma assistência personalizada, de acordo com as necessidades particulares de cada aluno. Para apoiar a analise desses desempenhos, este trabalho propõe uma abordagem estruturada como uma plataforma de suporte educacional baseado em jogos. Usando nossa abordagem, além dos benefícios promovidos pelos jogos educativos, fornecemos informações relevantes sobre o desempenho do aluno (erros comuns, simulação passo a passo, competências e habilidades, etc.), ajudando os professores em suas atividades acadêmicas.

\section{Introdução}

O uso de jogos na educação pode tornar os alunos mais engajados e comprometidos com os desafios educacionais [do Espírito Santo and de Menezes 2016]. Além das melhorias promovidas para a aprendizagem dos alunos, os jogos educativos também podem fornecer aos professores informações detalhadas sobre o desempenho dos alunos [Victal and de Menezes 2016]. Essa informação é importante, pois pode orientar os professores à ajustar seu planejamento e atender melhor as necessidades educacionais dos alunos. De fato, os jogos fornecem formas interessantes de avaliação, nas quais os alunos podem jogar sem necessariamente saber que estão sendo monitorados e avaliados [Novak et al. 2016].

Neste contexto, este trabalho propõe uma abordagem para facilitar o desenvolvimento de jogos educativos de forma que informações relevantes sobre o desempenho dos alunos, no jogo, possam ser registradas e analisadas. O objetivo é gerar dados para os professores sobre as deficiências acadêmicas desses alunos. Um exemplo de ferramenta 
VII Congresso Brasileiro de Informática na Educação (CBIE 2018)

Anais do XXIV Workshop de Informática na Escola (WIE 2018)

também é apresentado neste artigo, mostrando como a abordagem pode ser implementada em jogos educacionais reais.

Este artigo está organizado da seguinte forma: a próxima Seção contém a metodologia de pesquisa. Na Seção 3 apresenta-se trabalhos relacionados. A Seção 4 elucida nossa abordagem, mostrando seus componentes e modelos necessários para sua execução. A Seção 5 mostra a aplicação proposta. Por fim, na Seção 6 e 7 temos discussões levantadas e considerações finais.

\section{Metodologia de Pesquisa}

O principal objetivo dessa ferramenta é apoiar o professor, fornecendo ferramentas para que ele consiga visualizar e estruturar uma avaliação de conhecimentos dos alunos com base em dados gerados a partir de jogos digitais. Para isso, fez-se necessário conhecer o estado da arte e aprofundar sobre o conteúdo e trabalhos, uma revisão da literatura fezse necessária à procura de elementos centrais como: jogos digitais, avaliação, design de jogos e motores de jogos. Com isso identificou-se os trabalhos relacionados presentes na seção 3, cujos quais ajudaram a ter outra perspectiva e reflexão sobre problemas.

Encontrou-se uma quantidade relevante de jogos que auxiliam no ensino aprendizagem, porém quando analisados quais davam suporte a avaliação e tinham um feedback tanto para o aluno, quanto para o professor, nota-se uma escassez. A partir desse ponto, fez-se necessário pesquisar quais informações, da interação do aluno com o conteúdo educacional inserido em um jogo, são relevantes para os professores e como essas informações poderiam ser apresentadas. Para isso, professores foram entrevistados para elucidar esses questionamentos.

Com esses dados, realizamos o processo de analise e desenvolvimento da ferramenta, com o uso de diagramas UML para a modelagem, concepção da arquitetura e aplicação para que se torne viável uma validação preliminar com alunos e professores, bem como a elaboração dos modelos que apresentam as informações com relação a interação do aluno com o conteúdo educacional inserido do jogo.

\section{Trabalhos Relacionados}

Na literatura, existem pesquisas desenvolvidas para apoiar a construção de jogos que promovam a avaliação do desempenho dos alunos. Algumas iniciativas baseadas em Design Centrado em Evidência (ECD) é a avaliação furtiva proposta em [Shute 2011] e [Shute and Ventura 2013]. Os dados de desempenho dos alunos são continuamente coletados durante a execução de um jogo, usando modelos para representam tarefas a serem realizadas no jogo e suas respectivas competências. Para quantificar a avaliação educacional, esse modelo é baseado em argumentos probabilísticos [Kim and Shute 2015]. Embora essas abordagens sejam interessantes, elas são conceituais e não detalham como implementar o ambiente necessário para suporta-las. O relatório de desempenho é gerado por uma ferramenta que necessitou a inserção manualmente dos dados por um especialista.

O AvaGames é uma aplicação web para a criação de Arquiteturas Pedagógicas baseadas em jogos digitais, que promove desenvolvimento das habilidades cognitivas e das experiências sociais e epistemológicas dos jogadores [Victal and de Menezes 2016]. 
VII Congresso Brasileiro de Informática na Educação (CBIE 2018)

Anais do XXIV Workshop de Informática na Escola (WIE 2018)

Já [Pereita and Bonini-Rocha 2013] propôs um ambiente para capacitação por meio de jogos que misturam Quiz com Batalha Naval, cujo objetivo de imergir os jovens na experiência de aprendizagem, motivando alunos desmotivados. Um ponto negativo é que tais trabalho não exploram/informam a existência de relatórios fornecidos para o professor visualizar o desempenho do aluno.

\section{Game-Based Educational Software (GBES)}

A abordagem proposta consiste em uma plataforma e software educacional baseada em jogos (um jogo e um conjunto de relatórios de desempenho), como mostrado na Figura1. A plataforma GBES (Game-Based Educational Software) é um servidor Web que fornece um conjunto de serviços Web consumidos por um ou mais softwares e jogos.

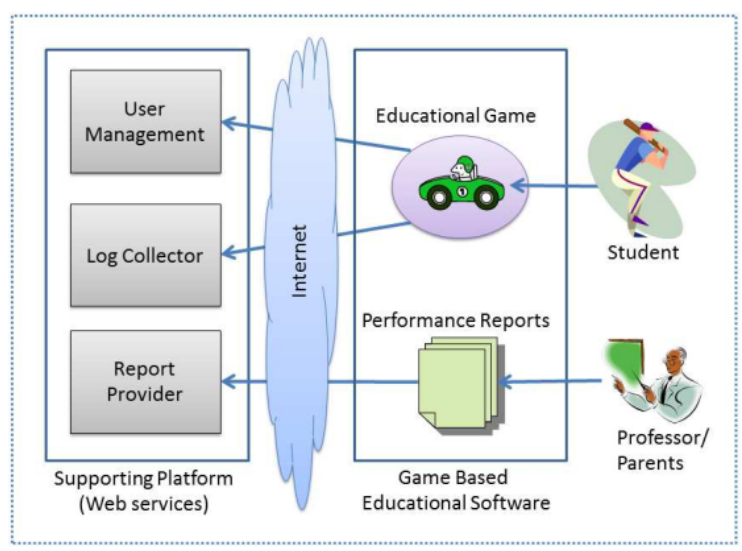

Figure 1. Principais componentes da abordagem proposta.

O GBES é estruturado com o uso de jogos educativos e um conjunto de relatórios de desempenho específicos relacionados à esses jogos. Esses dois principais componentes da abordagem são detalhados a seguir. O principal objetivo da plataforma, (Supporting Platform Figura 1), é oferecer suporte ao gerenciamento de usuários, coleta de logs e geração de relatórios. O componente User Management é usado para identificar alunos, professores e pais. Por sua vez, o componente Log Collector é responsável por receber e armazenar os eventos relevantes ocorridos durante o jogo. Por outro lado, o componente Report Provider fornece interfaces de consulta para os dados registrados.

Com relação ao componente Log Collector, todas as mensagens de log contêm o registro de data e hora do evento a ser registrado, a identificação do usuário que está executando o jogo (user_id) e o próprio jogo (game_id). O tipo de log (type) categoriza as mensagens de acordo com cada tipo de evento (início ou fim do jogo, resposta correta ou incorreta, etc.). O dados de log (data) contém os detalhes sobre o evento registrado. $\mathrm{O}$ formato dos dados do registro pode variar de acordo com o definido do jogo e o tipo de registro, usado pelo mecanismo de geração de relatórios do GBES. Essa característica permite que uma implementação de plataforma, suporte a diferentes jogos.

O componente Report Provider é responsável analisar as mensagens de log para gerar relatórios de desempenho. Esse componente filtra (por user_id, session_id, type, etc.) e acessa mensagens de log geradas pelos jogos. Com base nessas informações, relatórios podem ser gerados para mostrar o desempenho de um único aluno ou grupo 
VII Congresso Brasileiro de Informática na Educação (CBIE 2018)

Anais do XXIV Workshop de Informática na Escola (WIE 2018)

de alunos, ou até para simular as sessões realizadas por alunos nos desafios educacionais incorporados em um jogo.

Uma vez que o jogador é autenticado na plataforma, através de um cadastro, o jogo pode começar e todos os eventos e ações relevantes realizados pelo aluno são registrados na plataforma. Essas ações e eventos relevantes podem ser indicações de desempenho de sucesso ou fracasso durante os desafios educacionais do jogo.

Depois de jogar, os professores e/ou pais podem acessar os Relatórios de desempenho dos alunos. Esse componente do GBES é responsável por recuperar e filtrar todas as informações registradas, gerar relatórios que indicam o desempenho dos alunos, principais erros observados e etc. Para habilitar esses relatórios, é necessário criar os seguintes modelos:

1) Modelo de ação: Durante o design do jogo, é importante identificar eventos e ações do usuário que sejam relevantes para avaliar os conhecimentos, habilidades e competências do jogador.

2) Modelo de desempenho básico: esse modelo define relatórios que permitem a observação do envolvimento dos alunos e o desempenho educacional detalhado. Os relatórios geralmente são genéricos, independentemente do design do jogo. Por exemplo, alguns deles podem mostrar com que frequência um aluno joga um determinado jogo, por quanto tempo e etc.

3) Modelo de simulação: Alguns alunos podem precisar de uma atenção adicional dos professores, que devem ter entendimento de como esses alunos estão pensando para melhor apoiá-los. O modelo de simulação é usado para mostrar (passo a passo) das ações relevantes para a aprendizagem feitas por cada aluno em um determinado jogo.

4) Modelo de proficiência: O objetivo deste modelo é representar as competências, habilidades e conhecimentos dos alunos a serem avaliados. Este modelo deve ser criado durante o design do jogo, com base nos objetivos educacionais do jogo. Modelos probabilísticos podem ser empregados para representar a incerteza de proficiência.

5) Modelo de medição de aprendizagem: os indicadores de proficiência de competências, habilidades e conhecimentos devem ser medidos com base nas ações dos alunos no jogo. Este modelo é responsável por transformar dados brutos em um tipo de indicador. As regras de informação devem ser definidas para analisar respostas e ações definidas no modelo de ação. A saída dessas regras são evidências sobre a proficiência do aluno.

\section{Abordagem em Ação}

Nesta seção, apresentamos a aplicação da abordagem proposta em um jogo educacional.

\subsection{Game Design}

Esta seção descreve o componente do GBES, o jogo Trenzinho Numérico desenvolvido neste estudo para ajudar na compreensão da aplicação nossa abordagem, bem como na realização dos testes. Sua tela inicial (veja a Figura 2) apresenta um trem, seus vagões, alguns números e o herói do jogo. Cada vagão acomoda o número correspondente sua posição do vagão no trem. Dessa forma, o jogo trabalha conceitos básicos de matemática: identificação e ordenação números. 


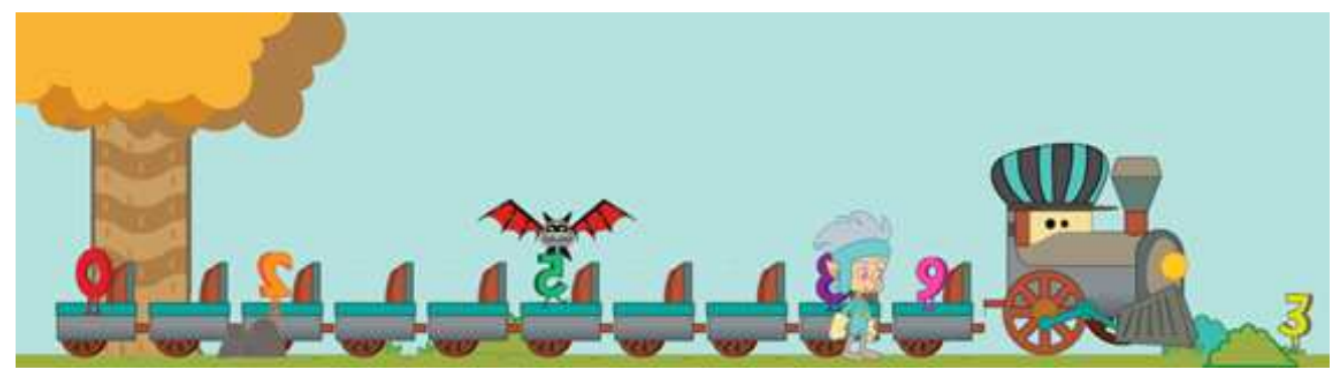

Figure 2. Tela inicial do jogo considerado no estudo de caso.

Antes de começar o jogo, alguns dos números escaparam dos vagões e o herói tem que encontrar, capturar e colocar cada número perdido, que estão espalhadas pela fase, em seus respectivos vagões. O número de vagões e números em falta varia em cada sessão de jogo, de acordo com as fases jogadas pelo aluno. Uma vez que o herói capture um número perdido, ele deve posicionar o número no vagão correto, que pode ser identificado com base na posição dos outros números no trem/vagões. Se o herói tentar posicionar um determinado número em um vagão errado o número escapa do vagão, destacando o erro do aluno. Se o herói posiciona um número perdido em seu vagão correto, o número permanece lá, destacando seu sucesso. Finalmente, quando todos os vagões estão cheios de números, o trem sai do cenário e a sessão de jogo termina.

Para testes preliminares, realizou-se sessões com 5 alunos (dois meninos e três meninas) entre quatro e seis anos de idade, equivalente a segundo ano da Educação Infantil e Primeiro ano do Ensino Fundamental, ambos os alunos por possuírem o habito de jogar, já estavam familiarizados com a tecnologia. O jogo foi aplicado por cerca de 40 minutos, capturando dessa forma, uma quantidade razoável de registros gerados pela interação dos alunos. A seguir, veremos os modelos, descritos na sessão 4, que foram geradas para esses alunos.

\subsection{Modelos Gerados}

A seguir, veremos quais os modelos que foram gerados para realização da captura das informações, simulação, proficiência e medição da aprendizagem do aluno. Tais modelos são encontrados na área de relatório das turmas no perfil do professor ou na área dos jogos no perfil dos alunos.

\section{Modelo de Ação}

Durante o design do jogo, todos os eventos e ações relevantes para analisar o desempenho do aluno foram identificados: I - início e fim do jogo (data e hora); II - Sucesso e falha na ordem de um determinado número, informando qual número foi colocado em um determinado vagão. Com base nessa lista, o desenvolvedor do jogo deve codificar para gerar mensagens JSON para registrar cada instância de eventos geradas pelo usuário.

Para cada tentativa de completar o trem, um número aleatório é gerado para o id da sessão. O tipo do $\log$ é 1 , representando um log de falhas: uma tentativa de posicionar um número no vagão vazio errado. Finalmente, o campo de dados informa o vagão e o número relacionado a essa ação. Neste caso, o herói tentou posicionar o número 6 no vagão do número 7 , configurando um erro. 
VII Congresso Brasileiro de Informática na Educação (CBIE 2018)

Anais do XXIV Workshop de Informática na Escola (WIE 2018)

\section{Modelo Básico de Desempenho}

Relatórios de desempenho com diferentes níveis de detalhes de informação foram criados neste estudo. O primeiro gráfico apresentado na Figura 3, mostrando uma visão geral dos erros, Figura 3, durante as sessões do jogo. O gráfico indica com que frequência um número não foi ordenado corretamente e o total de erros cometidos. Na Figura 3, o número 8 foi posicionado 20 vezes incorretamente pela turma. Este gráfico pode ser gerado para um aluno específico ou para todos os alunos que jogaram o jogo. Para fazer isso, o relatório chama um serviço da web solicitando todos os registros registrados do tipo 1 (erro).

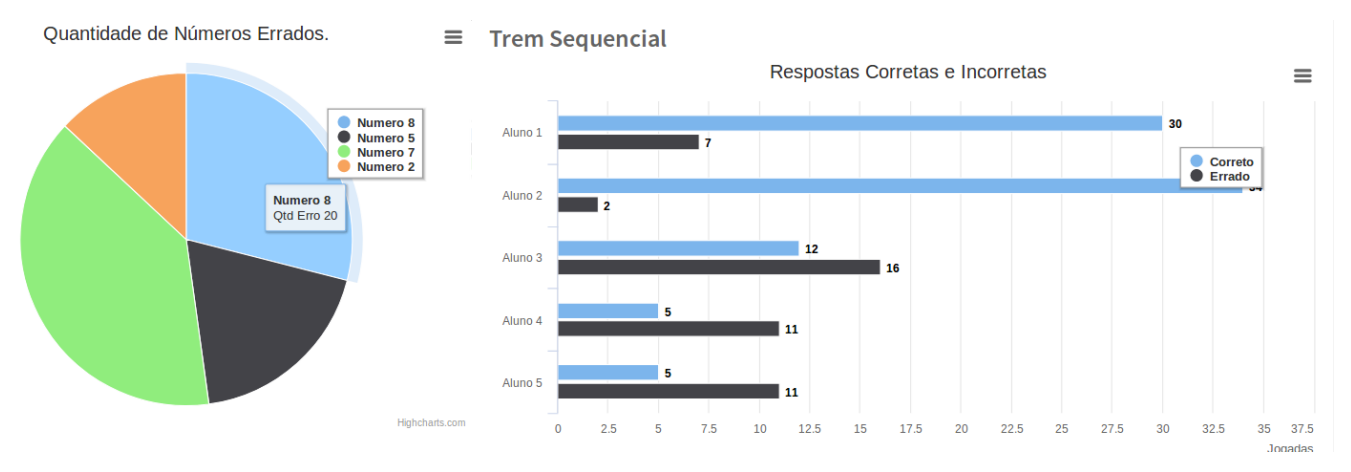

Figure 3. Frequência de erros por número relacionado.

O segundo gráfico, Figura 3, mostra uma lista dos estudantes e seus respectivos desempenhos no jogo. A lista mostra para cada aluno o número de tentativas de posicionar um número em um vagão e a porcentagem de tentativas corretas (barra azul) e incorretas (barra preta). Esta informação é calculada com base nos registros dos tipos 0 e 1.

Se o professor quiser ver o desempenho de um aluno em especifico, para ter uma visão mais detalhada, basta clicar em seu nome. Em seguida, ele acessará as informações apresentada na Figura 4. Este é um relatório mais detalhado, indicando o número de sessões de jogo e a evolução do desempenho em todas elas. Finalmente, o professor pode observar uma determinada sessão de jogo para entender melhor as dificuldades de seus alunos. A Figura 5 mostra um exemplo de como a informação é apresentada ao professor. É uma visão simplificada do jogo, simulando uma versão impressa do exercício. Os números em preto são números fixados em seus vagões. Aqueles em verde $(0,4$ e 7$)$ foram posicionados corretamente pelo aluno. $\mathrm{O}$ vermelho mostra um erro cometido pelo aluno (posicione o 0 no lugar de 4). Os logs do tipo 3 e 1 são usados para criar este relatório.

\begin{tabular}{|l|l|l|l|}
$\begin{array}{l}\text { Sessões: } \\
\text { DATE }\end{array}$ & NíveL & PERFORMANCE & SIMULATOR \\
\hline 23 de Fevereiro de 2017 & Medium & $70 \%$ & Visuallzar \\
\hline 24 de Fevereiro de 2017 & Low & $100 \%$ & Visuallizar \\
\hline 26 de Fevereiro de 2017 & High & $81,3 \%$ & Visualizar \\
\hline
\end{tabular}

Figure 4. Lista de sessões de jogo de um determinado aluno.

A Figura 5 apresenta o relatório de ações do aluno ao longo do tempo, mostrando 
VII Congresso Brasileiro de Informática na Educação (CBIE 2018)

Anais do XXIV Workshop de Informática na Escola (WIE 2018)

suas ações e decisões tomadas durante o jogo. É possível, por exemplo, observar a estratégia criada pelo aluno para ordenar os números, como e quando os erros ocorreram, etc. Para isso, os $\log s$ dos tipos 0 e 1 são usados e ordenados pelo timestamp.

\begin{tabular}{|l|l|l|l|l|l|l|l|l|l|}
\hline 0 & 1 & 2 & 3 & 4 & 5 & 6 & 7 & 8 & 9
\end{tabular}

Figure 5. Visualização simplificada do resultado do jogo.

\section{Modelo de Simulação}

Para uma compreensão mais profunda do comportamento do aluno, o professor pode simular as ações realizadas pelo aluno durante o jogo, como mostrado nas imagens presentes na Figura 6. A simulação mantém a visão simplificada proposta na Figura 5, com botões (iniciar, parar, avançar, pausar e rebobinar a simulação) para visualização do ações do aluno, passo a passo.

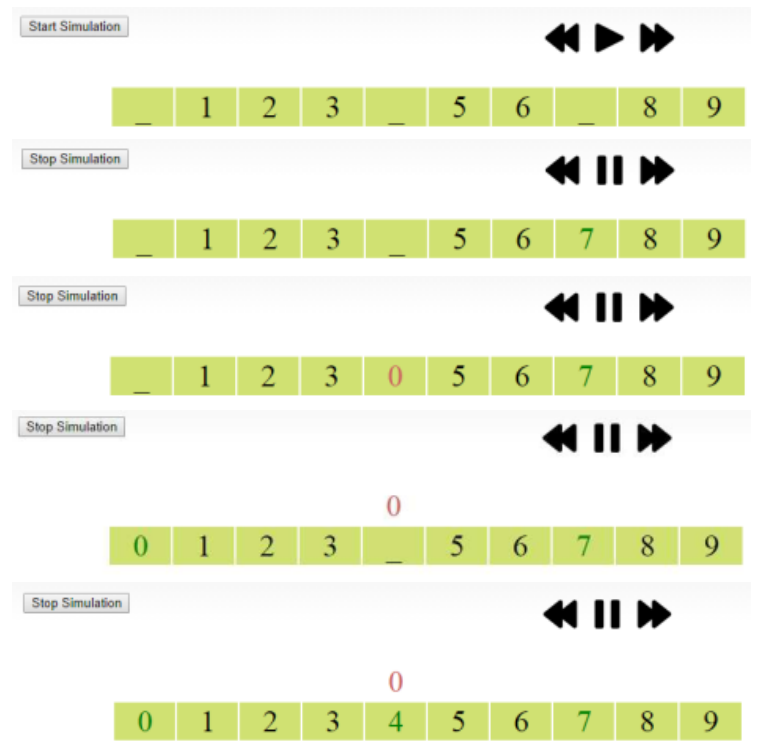

Figure 6. Tela de simulação inicial para uma dada sessão de jogo.

Na Figura 6 vemos uma sequencia da simulação: I - na primeira parte vemos a tela de simulação inicial para uma dada sessão de jogo; II - Posicionamento número 7 no lugar correto, III - Posicionando o número $0 \mathrm{em}$ um local incorreto; IV - Posicionando o número 0 no lugar correto; $\mathrm{V}$ - Posicionando o número 4 no lugar correto.

\section{Modelo de Proficiência}

Para este modelo, as duas habilidades matemáticas foram identificadas pelos desenvolvedores e professores: identificação de números e ordenação de números. Três níveis de proficiência foram definidos para cada um dos indicadores de habilidades, conforme apresentado nas Tabelas 1. Para cada nível de proficiência, uma probabilidade é calculada, representando a chance de um aluno ter esse nível de proficiência.

\section{Modelo de Medição de Aprendizagem}

O modelo de proficiência exige que as respostas corretas e incorretas sejam analisadas como evidências sobre a identificação do número e as habilidades de ordenação. 
VII Congresso Brasileiro de Informática na Educação (CBIE 2018)

Anais do XXIV Workshop de Informática na Escola (WIE 2018)

Table 1. Proficiência avaliada para ordenação de números e identificação de números de um aluno.

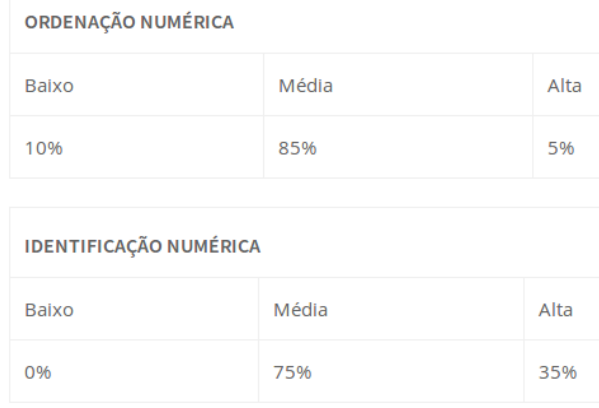

Para isso, as regras de transformação apresentadas na Tabela 2 são aplicadas.

Table 2. Regras para Ponto de ganho após uma resposta Correta e Incorreta.

\begin{tabular}{|l|c|c|c|}
\hline \multirow{2}{*}{ Competências } & \multicolumn{3}{|c|}{ Pontos Ganhos } \\
\cline { 2 - 4 } & 0 & 1 & 2 \\
\hline $\begin{array}{l}\text { Ordenação } \\
\text { Numérica }\end{array}$ & $\begin{array}{c}\text { Apenas um vagão } \\
\text { vazio }\end{array}$ & $\begin{array}{c}\text { Dois ou três vagões } \\
\text { vazios }\end{array}$ & $\begin{array}{c}\text { Mais de três vagões } \\
\text { vazios }\end{array}$ \\
\hline $\begin{array}{l}\text { Identificacãa } \\
\text { Numérica }\end{array}$ & $\begin{array}{c}\text { Apenas um vagão } \\
\text { vazio }\end{array}$ & $\begin{array}{c}\text { Dois ou mais vagões } \\
\text { vazios. }\end{array}$ & - \\
\hline
\end{tabular}

\begin{tabular}{|l|c|c|c|}
\hline \multirow{2}{*}{ Competência } & \multicolumn{3}{|c|}{ Pontos Perdidos } \\
\cline { 2 - 4 } & 0 & 1 & 2 \\
\hline $\begin{array}{l}\text { Ordenação } \\
\text { Numérica }\end{array}$ & - & $\begin{array}{c}\text { Mais de três vagões } \\
\text { vazios }\end{array}$ & $\begin{array}{c}\text { Dois ou três vagões } \\
\text { vazios }\end{array}$ \\
\hline
\end{tabular}

Cada resposta correta é uma evidência de ordem numérica e identificação de número. A força da evidência depende do contexto da resposta. Por exemplo, de acordo com a Tabela 2, se uma resposta correta coloca um dado número no último vagão vazio, ele tem peso zero (não foi necessário pensar). Quando dois vagões estão vazias no trem, um ponto é dado para as habilidades de ordenação de números e identificação de números. Quando há mais de três vagões vazios, é mais difícil colocar o número em ordem, então o peso da resposta é de dois.

Respostas incorretas também afetam o número de pontos no final de uma sessão de jogo. A Tabela 2 mostra o número de pontos perdidos para duas situações diferentes. Observe que todas as respostas incorretas reduzem um ou dois pontos da pontuação da sessão do jogo. No caso deste jogo, respostas incorretas são evidências contra apenas a proficiência de ordenação de números. Isso ocorre porque não é possível identificar o motivo de um erro, portanto, um erro de ordenação é considerado a causa raiz. Como consequência, outros jogos devem complementar a avaliação da proficiência de identificação de números.

Finalmente, após uma sessão de jogo, uma pontuação total é calculada com base no número de respostas corretas e incorretas. Uma escala de proficiência é determinada com base na pontuação máxima que pode ser obtida. Essa escala também é dividida em três partes, cada uma representando um nível de proficiência (alta, média e baixa). 
VII Congresso Brasileiro de Informática na Educação (CBIE 2018)

Anais do XXIV Workshop de Informática na Escola (WIE 2018)

Desta forma, cada sessão de jogo fornece uma evidência do nível de proficiência a ser considerado pelo modelo de proficiência.

\section{Discussão}

A Seção 5 apresentou uma aplicação da abordagem proposta, incluindo o desenvolvimento do GBES e a implementação da plataforma de apoio. O projeto foi apresentado e avaliado por três professores especialistas, um de ensino superior em computação e dois de matemática ensino fundamental e dois desenvolvedores de jogos e viram potencial na solução proposta.

Nesse curso foram levantados alguns conteúdos que são deficiências nos alunos. Apresentação de algumas atividades que são usadas em sala. Em seguida passou-se por um momento para elaborar game design de jogos visando trabalhar esse mesmo conteúdo e validando-os com os professores o aspecto pedagógico do jogo. Em seguida, definir o modelo de ação, proficiência e medição de aprendizagem.

Com esse contato viu-se a viabilidade da abordagem proposta, fornecendo uma maneira sistemática de adaptar ou criar jogos educativos para registrar informações e gerar relatórios de desempenho relevantes. De fato, os relatórios gerados foram apresentados e aprovados pelos professores que participam de um curso sobre o uso de jogos em sala de aula. Outra observação interessante durante este estudo foi o esforço para elaborar e implementar os relatórios de desempenho genéricos suficientes para que sejam inerentes ao tipo do jogo (Aventura, RPG, Puzzes e etc).

O planejamento do relatório deve ser realizado em conjunto com o design do jogo, uma vez que o design do jogo pode identificar ou melhor elaborar as ações relevantes em relação ao desempenho educacional. O número de relatórios diferentes que podem ser gerados é grande e deve ser avaliado, pois alguns relatórios podem levar um tempo significativo para serem implementados. Além disso, a análise de simulação pode ser realizada para entender melhor o pensamento e os erros dos alunos. Após esse encontro, houve o desenvolvimento de um dos jogos propostos e a execução da validação com os jogares presente na sessão 5 .

Existem limitações que devem ser consideradas em nossa análise. O primeiro é o jogo selecionado para o estudo. Nossa abordagem proposta parecia ser apropriada para jogos pequenos, mas pode exigir algumas adaptações para grandes jogos. Por exemplo, cada desafio em um jogo grande pode registrar suas informações e exigir relatórios específicos. Uma segunda limitação foi o tipo de análises realizadas no estudo. Nossa abordagem foi considerada apropriada para relatórios resumindo as ações relevantes dos alunos durante o jogo.

Vimos também uma limitação, devido ao fato da criação de jogos poderem ser de tipos, narrativas e design diversos a quantidade de tipos de relatórios e de modelos de simulação se torna um fator limitador, fazendo com que o game design desenvolva sua soluções alinhadas aos aos relatórios e ao modelo de simulação existentes na ferramenta.

\section{Considerações Finais}

Jogos educativos têm muito a contribuir para o processo de aprendizagem e para aumentar o conhecimento dos professores em relação aos seus alunos. Este trabalho contribuiu com 
VII Congresso Brasileiro de Informática na Educação (CBIE 2018)

Anais do XXIV Workshop de Informática na Escola (WIE 2018)

a definição de uma infraestrutura para apoiar o desenvolvimento de jogos educativos e relatórios de desempenho, de forma que o desempenho dos alunos possa ser registrado e analisado. Os relatórios podem apresentar informações sobre o desempenho de um determinado aluno ou de um grupo deles. Isso é também possível simular as etapas de todos os alunos realizadas no jogo educativo.

O alinhamento com professores e desenvolvedores se mostrou bastante proveitoso pois podemos notar alguns aspectos pedagógicos que não havíamos pensados e o alinhamento deles como o desenvolvimento do game design do jogo, consequentemente seu desenvolvimento.

Como trabalho futuro, pretendemos realizar mais estudos para verificar outros aspectos, como quão geral e aplicável é a nossa abordagem, e também quão difícil é desenvolver jogos usando nossa abordagem. Outro ponto sugerido pelos professores é o de analisar os comportamentos dos alunos para encontrar padrões nas ações dos mesmos, afim de inferir algum tipo de informação, como por exemplo, descobrir se os alunos que estão com mais dificuldades possuem ações em comum e etc. Além disso, mais estudos devem ser realizados para avaliar quão apropriada é a nossa abordagem para diferentes tipos de jogos educacionais, ou quão caro é usar nossa abordagem.

\section{References}

Chodorow, K. (2013). MongoDB: The Definitive Guide: Powerful and Scalable Data Storage. " O’Reilly Media, Inc.".

do Espírito Santo, D. and de Menezes, C. (2016). Um ambiente para autoria e realização de aventuras pedagógicas digitais. In Anais dos Workshops do Congresso Brasileiro de Informática na Educação, volume 5, page 588.

Kalin, M. (2013). Java Web Services: Up and Running: A Quick, Practical, and Thorough Introduction. " O'Reilly Media, Inc.".

Kim, Y. J. and Shute, V. J. (2015). Opportunities and challenges in assessing and supporting creativity in video games. In Video games and creativity, pages 99-117. Elsevier.

Novak, E., Johnson, T. E., Tenenbaum, G., and Shute, V. J. (2016). Effects of an instructional gaming characteristic on learning effectiveness, efficiency, and engagement: using a storyline for teaching basic statistical skills. Interactive Learning Environments, 24(3):523-538.

Pereita, J. L. D. and Bonini-Rocha, A. C. (2013). Prática de informática na educação: Ambiente de experiência itae com alunos da universidade de brasília (unb). In Anais dos Workshops do Congresso Brasileiro de Informática na Educação, volume 2.

Shute, V. J. (2011). Stealth assessment in computer-based games to support learning. Computer games and instruction, 55(2):503-524.

Shute, V. J. and Ventura, M. (2013). Measuring and supporting learning in games: Stealth assessment.

Sriparasa, S. S. (2013). JavaScript and JSON essentials. Packt Publishing Ltd.

Victal, E. and de Menezes, C. (2016). Avagames: Um ambiente para apoio à avaliação da aprendizagem em jogos digitais. In Anais dos Workshops do Congresso Brasileiro de Informática na Educação, volume 5, page 477. 